\title{
New Methods of Preparing Cross Sectional Samples and Ultra-Thin Foils for SEM and TEM by a Broad Argon Ion Beam
}

\author{
K. Ogura ${ }^{1}$, N. Erdman ${ }^{2}$, A. R. Campbell ${ }^{2}$, and S. Asahina ${ }^{1}$ \\ ${ }^{1}$ JEOL Ltd. 1-2 Musashino 3-chome. Akishima, Tokyo 196-8558 Japan \\ ${ }^{2}$ JEOL USA, Inc., Peabody, MA \\ Correspondence: kogura@jeol.co.jp
}

Currently various methods are used to prepare specimen cross sections for SEM. Mechanical methods of cutting and polishing are widely used. However, cutting or mechanical polishing of composite materials with different hardness requires sophisticated skill. An ultra microtome and FIB (Focused Ion Beam) are also used to prepare cross sections of specimens. The ultra microtome is mainly used for preparation of biological and polymer specimens. The FIB is a powerful tool when precise positioning of a cross section is required. However, the size of the resulting cross section is very limited.

The new specimen preparation apparatus, Cross-section Polisher (CP), utilizes a broad argon ion beam which eliminates these difficulties associated with the conventional methods of specimen cross-sectioning for SEM(1)(2). The CP consists of a specimen chamber with a TMP vacuum system, an optical microscope for specimen positioning, and controllers of vacuum system and ion beam. The stage in the specimen chamber features a specimen holder and a masking plate. A region of interest to be cross sectioned is selected under an optical microscope. Then a masking plate is placed across the region. After evacuating the specimen chamber, the region is irradiated with a broad argon ion beam (Fig.1). Accelerating voltage range of the argon ion beam is 2 to $6 \mathrm{kV}$. The specimen stage can be rocked $\pm 30^{\circ}$ during the ion beam irradiation. Figs. $2 \mathrm{a}$ and $2 \mathrm{~b}$ show backscattered electron images of a cross-section of gold wire bonding on silicon prepared with the CP. In spite of the big difference in hardness between silicon and gold, the cross-section is very high quality, and clearly reveals narrow cracks and small voids. Channeling contrast in gold can also be clearly observed. It took 4 hours to make this cross section using an accelerating voltage of $6 \mathrm{kV}$. These images were taken with an FESEM (JSM-7000F).

Another new specimen preparation apparatus, Ion Slicer (IS), prepares ultra-thin foil specimens for TEM and STEM. A masking belt is employed in the IS instead of the masking plate in the $\mathrm{CP}$ (3). The masking belt is $10 \mu \mathrm{m}$ thick, and is placed on top of the specimen. An argon gun is mechanically rocking above the masking belt to mill the specimen from both sides of the masking belt (Fig.3). Rocking angle of the ion gun is up to $\pm 4^{\circ}$. The milled area size of the thin foil depends on the rocking angle of the ion gun. Smaller rocking angles result in wider areas of thin film. Both the 
specimen and the masking belt are rocking $\pm 30^{\circ}$ during the irradiation with the ion beam. Figs. $4 \mathrm{a}$ and $4 \mathrm{~b}$ show $200 \mathrm{kV}$ TEM images of a ceramic (SiAlON) prepared with the IS. It took 2 hours to make this ultra-thin foil using an accelerating voltage of $6 \mathrm{kV}$ with the rocking angle set at $\pm 2^{\circ}$. As a result of small rocking angle of the gun the prepared specimen had a wide electron-transparent area, as shown in a low magnification image of Fig.4a. The high-resolution TEM image is shown in Fig.4b.

With the CP and the IS, respectively, cross sections and ultra-thin sections of specimens can be prepared faster and easier than with conventional specimen preparation methods.

(1) M. Shibata, S. Asahina, T. Negishi, Proc. 8 APEM, (Kanazawa, 2004) p258

(2) Hauffe W, Electron Microscopy, Vol. 2, EUREM 92, Granada, Spain, 1992

(3) A. Yasuhara, JEOL News, Vol. 40 No. 1 (2005) p46-49.
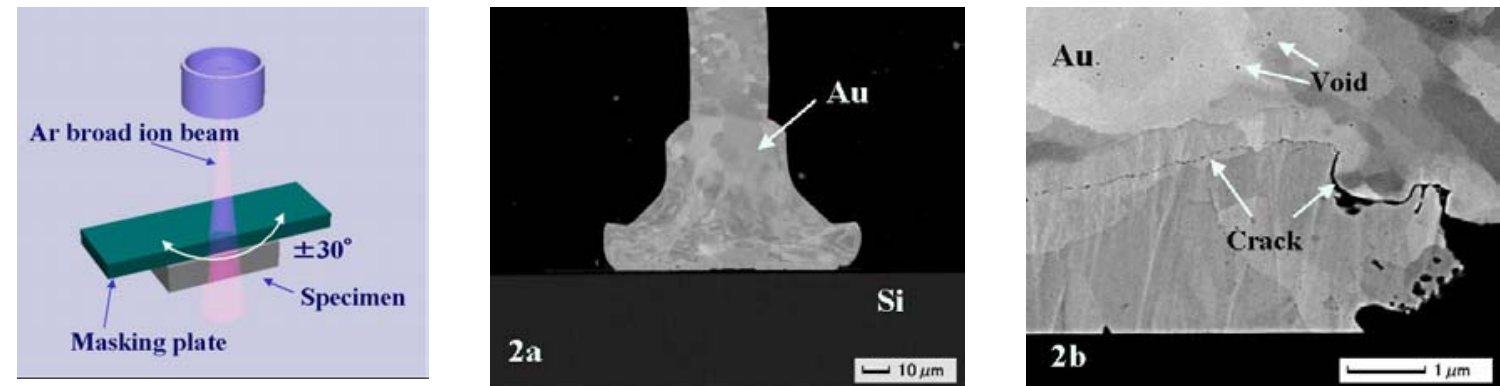

Fig.1 Principle of Cross Section Polisher (CP) Fig. 2 SEM images of a cross section of gold wire bonding on silicon
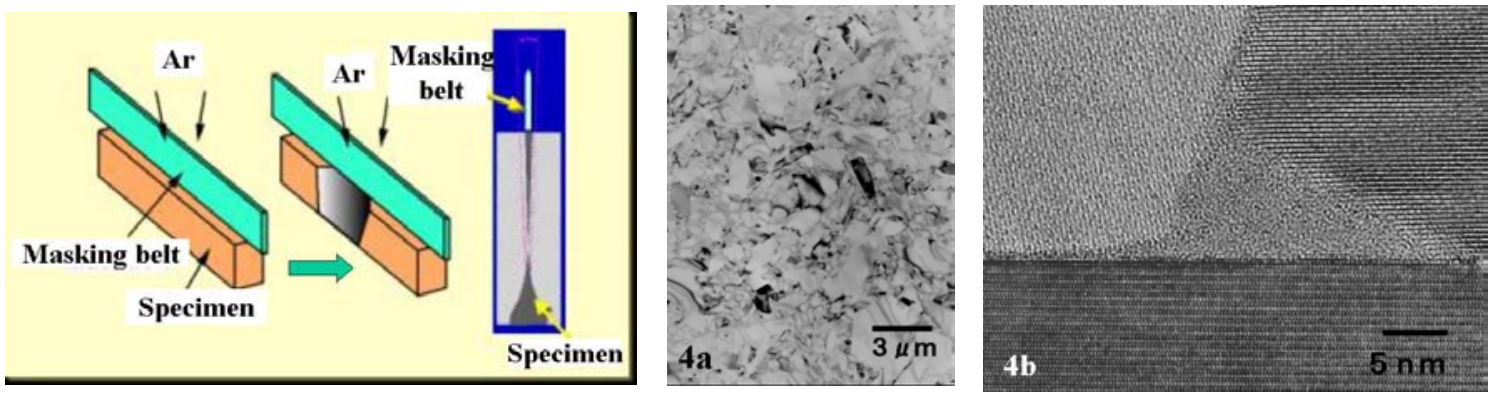

Fig.3 Principle of Ion Slicer (IS)

Fig.4 $200 \mathrm{kV}$ TEM images of a ceramic (SiAlON) 